\title{
Treatment failure and associated factors among first line patients on highly active antiretroviral therapy in Ethiopia: a systematic review and meta-analysis
}

\author{
Moges Agazhe Assemie ${ }^{1 *}$, Muluneh Alene ${ }^{1}$, Daniel Bekele Ketema ${ }^{1}$ and Selishi Mulatu²
}

\begin{abstract}
Background: Antiretroviral therapy (ART) restores immune function and reduces human immunodeficiency virus (HIV) related adverse outcomes. The results of previous studies in Ethiopia were replete with inconsistent findings; nonexistence of national representative figures and determinant factors are found as significant gap. The aim of this systematic review and meta-analysis was to assess the existing evidence on ART treatment failure and associated factors in Ethiopia.

Methods: Relevant studies on ART treatment failure were retrieved from international databases: PubMed, Google Scholar, Scopus, and Science Direct systematically prior to March 14, 2019. All identified studies reporting the proportion of first line treatment failure among HIV patients in Ethiopia were included. Two authors independently extracted all necessary data using a standardized data extraction format. A random-effects model was used to calculate pooled estimates and associated factors in Stata/se Version-14. The Cochrane Q test statistics and $P$ tests were used to assess the heterogeneity of the studies.

Results: From 18 articles reviewed; the pooled proportion of first line treatment failure among ART users in Ethiopia was $15.3 \%(95 \%$ Cl: $12,18.6)$ with $\left(P^{2}=97.9 \%, p<0.001\right)$. The subgroup analysis by World Health Organization $(\mathrm{WHO})$ treatment failure assessment criteria were carried out, accordingly the highest prevalence (11.5\%) was noted on immunological and the lowest (5.8\%) was observed virological treatment failure. We had found poor adherence $(\mathrm{OR}=8.6,95 \% \mathrm{Cl}: 5.6,13.4)$, not disclosed $(\mathrm{OR}=2.1,95 \% \mathrm{Cl}: 1.5,3.0)$, advanced $\mathrm{WHO}$ clinical stage III/IV $(\mathrm{OR}=2.4,95 \% \mathrm{Cl}: 1.5,3.8)$, change in regimen $(\mathrm{OR}=2.5,95 \% \mathrm{Cl}: 1.6,3.9)$ and being co-infected $(\mathrm{OR}=2.56,95 \% \mathrm{Cl}: 2.2,3.0)$ were statistically significant factors for treatment failure.

Conclusion: In this study, treatment failure among ART users in Ethiopia was significant. Adherence, co-infection, advanced WHO clinical stage, regimen change, and disclosure are determinant factors for treatment failure. Therefore, improve drug adherence, prevent co-infection, close follow up, and prevent HIV-drug resistance are required in future remedial efforts.
\end{abstract}

Keywords: Antiretroviral therapy, First-line treatment, Treatment failure, Switching to second-line, HIV/AIDS, Ethiopia

\footnotetext{
* Correspondence: agazhemoges@gmail.com

${ }^{1}$ Department of Public Health, College of Health Science Debre Markos

University, P.O. Box 269, Debre Markos, Ethiopia

Full list of author information is available at the end of the article
}

(c) The Author(s). 2019 Open Access This article is distributed under the terms of the Creative Commons Attribution 4.0 International License (http://creativecommons.org/licenses/by/4.0/), which permits unrestricted use, distribution, and reproduction in any medium, provided you give appropriate credit to the original author(s) and the source, provide a link to the Creative Commons license, and indicate if changes were made. The Creative Commons Public Domain Dedication waiver (http://creativecommons.org/publicdomain/zero/1.0/) applies to the data made available in this article, unless otherwise stated. 


\section{Background}

Ethiopia is one of the low-income countries experiencing high communicable disease burden, including HIV/AIDS which accounts 70 disability adjusted life years per 100, 000 individuals $[1,2]$. Access to highly active antiretroviral therapy (HAART) in Ethiopia started in 2005, and reached 420,000 people from 716, 418 people living with HIV/AIDS by 2016 ART [3, 4]. Even though ART is not a curative medicine, access to HAART has played a vital role in the clinical management of HIV- infected individuals through reestablishing the immune function and preventing morbidity and mortality. HAART also expected to contribute significant role to reduce new HIVinfection by $2020[5,6]$.

Even though many HIV-positive clients have accessed ART, first line treatment failure continues to grow in resource limiting countries. First-line antiretroviral treatment is a combination of two nucleoside reverse-transcriptase inhibitors plus a non-nucleoside reverse-transcriptase inhibitor while treatment failure is the progression of HIV infection after the initiation of ART $[7,8]$.

Failure can be assessed, based on WHO criteria, as clinical, immunologic, virologic or a combination. Regular treatment failure detection is low because of inadequate capacity and lack of laboratory facilities in resource-limited settings including Ethiopia [9].

Treatment failure is frequently linked to mortality, which is costly locally, and the development of drug resistant viral strains, which has global implications $[10,11]$. The imperative for compliance with lifelong use of medication to avert negative outcomes is a significant challenge [12-14]. Therefore, early detection of treatment failure is a key to sustain first-line therapy effectiveness and to prevent HIV-drug resistance [12, 13].

There are many studies conducted in Ethiopia to determine the prevalence of first line treatment failure [15-31]. However, the magnitudes of these studies were inconsistent and characterized by great variability: nonexistence of country wide data to represent national treatment failure and its determinant factors are identified as significant gap. The aim of this systematic review and meta-analysis was to estimate the pooled prevalence and associated factors of treatment failure in the Ethiopia. This finding will assist decision makers and other concerned stakeholders to design, implement and evaluate effective and efficient interventions to improve the ART adherence in order to decrease morbidity, mortality, and development of drug resistance.

\section{Methods}

\section{Study design and settings}

Systematic review and meta-analysis using computerized databases; searches were performed to locate all studies to estimate the pooled prevalence of treatment failure in
HIV/AIDS patients in Ethiopia which is found in east Africa.

\section{Search strategy}

To find potentially relevant articles, a comprehensive search was carried out on Pub Med/MEDLINE, Google Scholar, Scopus and Science Direct databases. We extended our search by retrieving and extracting potential articles from reference lists of eligible articles as well as hand searching for grey literature and other relevant literature collections. The search of the literature was conducted between April 2018 and March 2019. All papers published prior to March 14, 2019 were considered. The search protocol was formulated by using common key words 'prevalence OR magnitude AND associated factor OR first-line OR switching OR second-line OR treatment failure AND antiretroviral therapy OR HIV/AIDS OR resourcelimited setting AND 'Ethiopia'. We followed and presented this meta-analysis according to the Preferred Reporting Items for Systematic Reviews and MetaAnalysis (PRISMA) guidelines [32].

\section{Eligibility criteria \\ Inclusion criteria}

Study area All studies conducted in Ethiopia were included in the systematic review and a combination of two and above studies used in the meta-analysis to produce single estimate of common effect.

Study design All observational study designs reporting the prevalence of first line treatment failure.

Population All HIV positive patients on first line antiretroviral treatment.

Language Only articles reported in English language.

Publication condition Published and unpublished articles.

Exclusion criteria Articles, which were not fully accessed or when they are not fully explain first line treatment failure. Exclusion of these articles is because of the inability to assess the quality of articles in the absence of full text.

\section{Outcome variables}

This study has an outcome variable prevalence of first line treatment failure, which is defined as clinical, immunological, virological or some combination of those outcomes [7] and determinant factors. We determine the association between treatment failure and associated factors in the form of the log odds ratio. The determinants included in this review were adherence, advanced 
WHO stage, CD4 count, regimen change, functional status, co-infection and disclosure.

\section{Data extraction}

All identified studies were screened via title and abstract for inclusion by two reviewers (MA and DBK) independently extracting all necessary data. Discussions and mutual consensus processes were undertaken when disagreements were raised between the two reviewers. These reviewers then assessed the full text of potentially eligible papers. The primary author of the original research was contacted for additional information or to clarify method details as needed. The data extraction format included primary author, publication year, regions of the country, study area, sample size and prevalence with 95\% CI.

\section{Quality appraisal}

The included articles were evaluated for quality, with only high-quality studies included in the analysis. Two authors (MAA and MA) independently assessed the quality of each included paper using an adapted Newcastle-Ottawa Scale quality assessment tool for cross-sectional and retrospective cohort studies [33]. The tool has three sections in general; the first section is graded out of five stars and considers the methodological quality of each original study. The second section deals with the comparability of the study cases or cohorts, with a possibility of two stars to be gained. The third section focus on the outcome and statistical analysis of each original study with a possibility of three stars to be given. The reviewers compared their quality appraisal scores and resolved any discrepancy before calculating the final appraisal score. Articles with a score of $\geq 6$ out of 10 scales were considered high quality, which, in this instance, reflected all eligible studies (i.e., none were eliminated at this stage).

\section{Data processing and analysis}

Data on study design, sample size, study population/age group, mid-study period and publication year were extracted in Microsoft Excel format, and then analysis was carried out using STATA/se Version 14 software. Heterogeneity among reported prevalence was assessed by computing $p$-values of Cochrane Q-test and $I^{2}$-statics [34]. The pooled prevalence of treatment failure was carried out with a random effects meta-analysis model, generating the pooled $95 \%$ confidence interval using the DerSimonian and Laird's method [35]. Sub group analysis was done to investigate how treatment failure varies across different sub-group of patients and to minimize the random variations between the point estimates of the primary study; subgroup analysis was done based on WHO treatment failure assessment criteria, study design and sample size. In addition, univariate meta-regression was undertaken to identify the possible source of heterogeneity. Publication bias was assessed by Egger's and Beggs' tests at 5\% significant level [36]. Point prevalence, as well as $95 \%$ confidence intervals, was presented in the forest plot format. In this plot, the size of each box indicated the weight of the study, while each crossed line refers to $95 \%$ confidence interval. For the second outcome, a log odds ratio was used to examine the association between determinant factors and treatment failure.

\section{Results \\ PRISMA flow chart}

We retrieved 345 articles regarding prevalence of treatment failure among ART users as identified in PubMed, Google Scholar, Scopus, and Science Direct. Of these initial articles, 209 articles were non-duplicates; of this, 127 articles were excluded after reviewing their titles and abstracts, and confirmed irrelevance to this review. Thus, 82 potential full text articles were assessed for eligibility, which resulted in further exclusion of 64 articles due to the study conducted in other countries, inaccessibility of the full text articles and outcome of interest not reported. Finally, 18 studies met the eligibility criteria and were included in the final metaanalysis. As shown in the follow chart of study selection process (Fig. 1).

\section{Characteristics of included studies}

As described in Table 1, these 18 studies were both retrospective cohort and cross-sectional study design published prior to March 14, 2019 was included. In the current meta-analysis, 22,849 study participants were represented by 18 studies. The prevalence treatment failure reported was between (4.1\%) [17] and (22.2\%) [15] and the sample sizes of the studies ranged from 225 [16] to 4809 [21].

\section{Meta-analysis of treatment failure in Ethiopia}

Prevalence of ART treatment failure in Ethiopia was 15.3\% (95\% CI: $12,18.6)$ by using the three WHO treatment failure criteria (immunological, clinical and Virological). A high heterogeneity was observed across the included studies $(\mathrm{I} 2=97.9 \%, p<0.001)$ (Fig. 2). Therefore, a random effect meta-analysis model was computed to estimate the pooled prevalence of treatment failure in Ethiopia. From this metaanalysis, the highest prevalence was $22 \%(95 \% \mathrm{CI}: 18,26)$ reported in a study by Assefa et al. [15], whereas the lowest prevalence of $4 \%$ was reported by Ayalew et al. [38]. To identify the possible sources of heterogeneity; different factors associated with it, such as the region of the country where the study was conducted, study population, publication year and sample sizes were investigated by using univariate meta-regression models, but none of these variables 


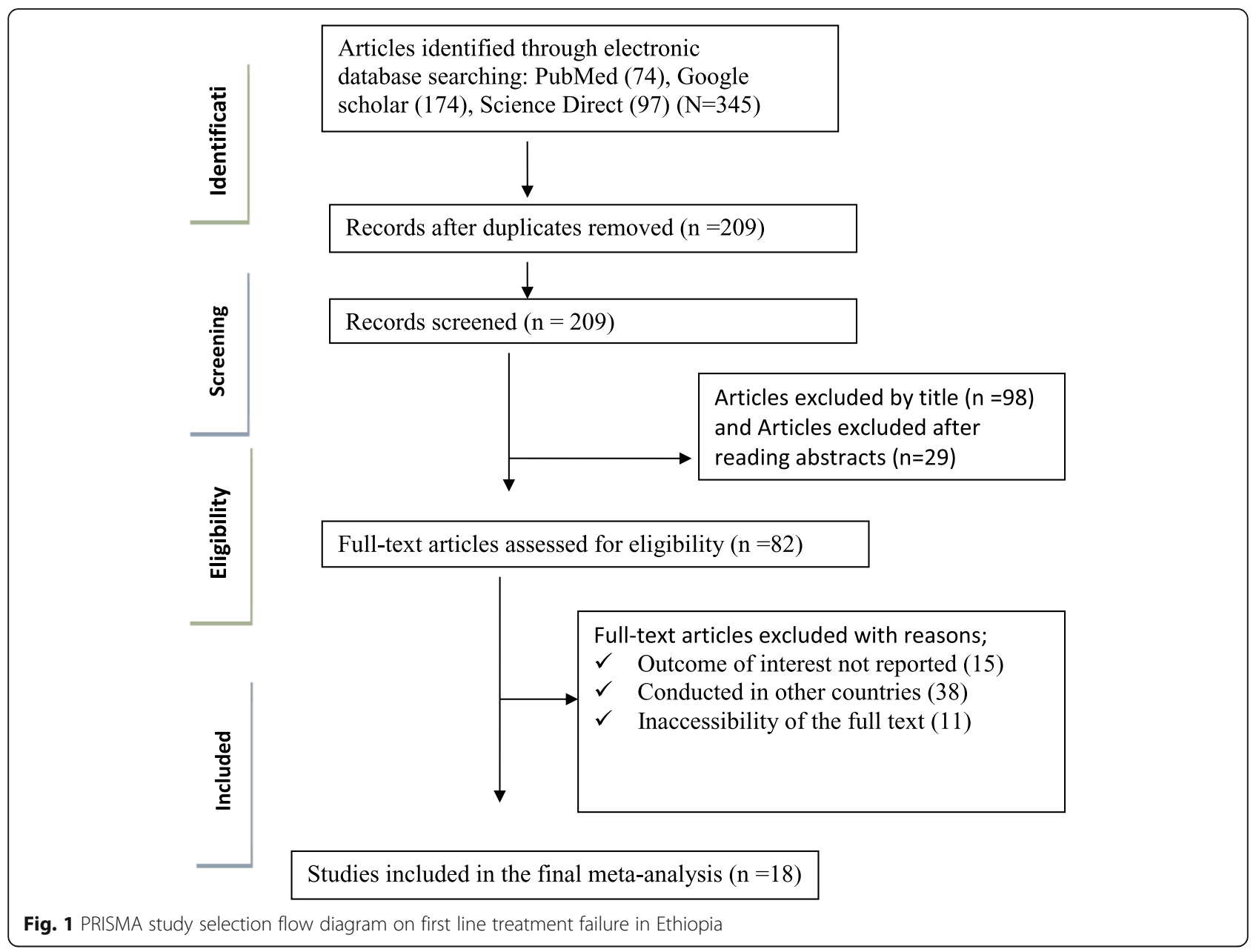

were found to be statistically significant (Table 2). We performed an objective based Publication bias assessment using Eggers' and Beggs' tests. Accordingly, those tests do not showed statistical significant for prevalence of treatment failure with $(p=0.80)$.

\section{Subgroup analysis}

In this meta-analysis, we computed subgroup analysis based on WHO failure assessment criteria, and the highest prevalence was observed by the immunological failure detection criteria (11.5\%) (95\%CI: 8.8, 14.3) and the lowest noted by virological confirmation at 5.8\% (95\% CI: $2.7,8.9$ ). We also carried out subgroup analysis on the study design yielding a prevalence in cross sectional of $15.9 \%$ (95\% CI: 9.2, 22.6) nearly the same in retrospective cohort $15.2 \%$ (95\% CI: 11.5, 18.9). As well, we also considered a subgroup analysis on sample size. Accordingly, the prevalence of first line treatment failure was higher in studies above mean (1269) samples $22 \%$ (95\% CI: $21.2,22.8$ ) compared to those with mean sample size below (1269) (15\%) (95\% CI: 11.8, 18.3).

\section{Associated factors of ART treatment failure in Ethiopia}

In this meta-analysis, we examined the association between ART drug adherence and treatment failure by using seven studies $[16,17,19,22,24,26,31]$. The findings from these seven studies revealed that the prevalence of treatment failure was significantly associated with adherence. Consequently, the probability of treatment failure was 8.6 times higher among patients who had missed appointments described by greater than 3 days per month (poor adherence) as compared to its counterpart (good adherence) $(\mathrm{OR}=8.6,95 \% \mathrm{CI}$ : 5.6, 13.4). The result of the statistics indicated that high heterogeneity ( $\mathrm{I} 2=92.5 \%$ and $p<0.001)$ was presented across the included studies (Fig. 3). Accordingly, a random effect meta-analysis model was performed to determine the association.

The association between co-infection and treatment failure based on four studies [16, 19-21] showed that the occurrence of treatment failure was associated with co-infection. Subsequently, the probability of treatment failure was 2.6 times higher among patients who had coinfection as compared to patients without co-infection 
Table 1 Descriptive summary of 18 studies included in the meta-analysis of the prevalence first line treatment failure in Ethiopia 2019

\begin{tabular}{|c|c|c|c|c|c|c|}
\hline Authorship & Publication (yr) & Study area /Region & Study population & Study period & Sample & Prevalence at $95 \% \mathrm{Cl}$ \\
\hline Hail et al. [21] & 2016 & Oromia & Adult & $2007-2014$ & 4809 & $9.4(8.6,10.2)$ \\
\hline Ayalew et al. [17] & 2016 & Amhara & Adult & 2011-2015 & 340 & $4.1(2.0,6.2)$ \\
\hline Sisay et al. [20] & 2017 & Addis Ababa & Adult & 2011-2016 & 595 & $21.5(18.2,24.8)$ \\
\hline Melsew et al. [30] & 2013 & Amhara & Adult & Jan-Apr,2007 & 509 & $21.0(17.5,24.5)$ \\
\hline Teshome et al. [27] & 2015 & Addis Ababa & Adult & 2009-2013 & 525 & $19.8(16.4,23.2)$ \\
\hline Yassin et al. [37] & 2017 & Oromia & Child & 2006-2015 & 269 & $18.8(14.1,23.5)$ \\
\hline Sisay et al. [23] & 2017 & Amhara & Child & 2010-2016 & 824 & $7.7(5.9,9.5)$ \\
\hline Bach et al. [25] & 2012 & Addis Ababa & Child & 2005-2011 & 1186 & $14.1(12.1,16.1)$ \\
\hline Yirdaw et al. [38] & 2015 & SNNP & Mixed & 2004-2012 & 1321 & $17.6(15.6,19.7)$ \\
\hline Assefa et al. [15] & 2014 & Amhara & Adults & 2007-2008 & 400 & $22.2(18.1,26.3)$ \\
\hline Teshome et al. [28] & 2015 & Addis Ababa & Adult & 2007-2009 & 293 & $15.7(11.5,19.9)$ \\
\hline Bekelech et al. [18] & 2015 & Oromia & Adult & 2006-2013 & 828 & $6.8(5.1,8.5)$ \\
\hline Brhane et al. [19] & 2017 & Amhara & Mixed & Aug-Sep, 2016 & 421 & $10.7(7.8,13.7)$ \\
\hline Zeleke et al. [16] & 2016 & Amhara & Child & Sept-Dec,2014 & 225 & $18.2(13.2,23.2)$ \\
\hline Agezew et al. [24] & 2019 & Amhara & Adult & 2012-2017 & 315 & $10(6.7,13.3)$ \\
\hline Hailu et al. [22] & 2018 & Tigray & Adult & 2008-2016 & 260 & $18(13.3,22.7)$ \\
\hline Getaneh et al. [31] & 2019 & National & Adult & 2016-2017 & 9284 & $22(21.2,22.8)$ \\
\hline Endebu et al. [26] & 2018 & Oromia & Adult & 2013-2018 & 445 & $9(6.3,11.7)$ \\
\hline
\end{tabular}

$(\mathrm{OR}=2.56,95 \% \mathrm{CI}: 2.2,3.0)$. The statistics indicated moderate heterogeneity $(\mathrm{I} 2=33.7 \%$ and $p=0.210)$ (Fig. 4). Therefore, a random effect meta-analysis model was executed to determine the association.

We observed the association between WHO clinical stage and treatment failure by using three studies [16, 24, 37]. The findings from these three articles revealed that the pooled prevalence of treatment failure was significantly associated with advanced WHO clinical stage. Thus, the likelihood of treatment failure was 2.4 times higher among patients who had advanced WHO clinical stage (III/IV) as compared to stage I and II (OR $=2.4,95 \%$ CI: $1.5,3.8)$. The result of the test statistics showed high heterogeneity (I2 = $85.5 \%$ and $p=0.001$ ) was presented across the articles (Fig. 5). Consequently, a random effect meta-analysis model was computed to determine the association.

The association between regimen change and treatment failure examined by using three studies $[16,25,26]$

\section{Study}

ID

Fig. 2 Forest plot of pooled prevalence on first line treatment failure in Ethiopia

ES (95\% CI)

$22.00(21.16,22.84)$ $10.00(6.69,13.31)$ $9.00(6.34,11.66)$ $18.00(13.33,22.67)$ $22.00(18.67,25.33)$ $8.00(6.15,9.85)$ $19.00(14.31,23.69)$ $20.00(16.18,23.82)$ $18.00(12.98,23.02)$ $4.00(1.92,6.08)$ $9.00(8.19,9.81)$ $20.00(16.58,23.42)$ $7.00(5.26,8.74)$ $16.00(11.80,20.20)$ $18.00(15.93,20.07)$ $22.20(18.13,26.27)$ $21.00(17.46,24.54)$ $14.00(12.03,15.97)$ $15.29(11.96,18.62)$

Overall (I-squared $=97.9 \%, p=0.000$ )

NOTE: Weights are from random effects analysis

on 10 
Table 2 Related factors with heterogeneity of treatment failure among ART users based on univariate meta-regression

\begin{tabular}{lll}
\hline Variables & Coefficient & $P$-value \\
\hline Publication year & -0.33 & 0.62 \\
Sample size & 0.001 & 0.3 \\
Region & & \\
$\quad$ Amhara & -3.9 & 0.57 \\
$\quad$ Addis Ababa & -0.001 & 0.99 \\
$\quad$ Oromia \& SNNP & -6.3 & 0.4 \\
Study Population & & \\
$\quad$ Adult & 0.6 & 0.9 \\
$\quad$ Child & 0.24 & 0.96 \\
\hline
\end{tabular}

revealed the pooled prevalence of treatment failure was associated with regimen change. Therefore, the chance of treatment failure was 2.5 times higher among patients who had regimen change as compared to their counterparts not changing their regimens $(\mathrm{OR}=2.5,95 \% \mathrm{CI}$ : 1.6, 3.9). The result of test statistics indicated that high heterogeneity ( $\mathrm{I} 2=86.2 \%$ and $p=0.001$ ) (Fig. 6). Hence, a random effect meta-analysis model was used to determine the association with the outcome.

Similarly, the association between discloser and treatment failure based on four studies [18, 23, 31, 37] showed that the prevalence of treatment failure was associated with disclosure. Therefore, the possibility of treatment failure was 2 times higher among patients who had not disclosed as compared to disclosed (OR = 2.1, 95\% CI: $1.5,3.0)$. The result of test statistics indicated high heterogeneity (I2 $=89.3 \%$ and $p<0.001)$
(Fig. 7). Therefore, a random effect meta-analysis model was used to determine the association with the outcome.

Finally, we examined the association between treatment failure and CD4 count as well as functional status with six and three studies respectively. However, both of these factors were not statistically associated with treatment failure.

\section{Discussion}

Treatment failure is one of the causes of mortality and development of drug resistant viral strains potentiating a significant challenge globally $[10,11]$. To the best of our knowledge, this meta-analysis is the first of its kind to estimate the pooled prevalence of treatment failure in Ethiopia.

The overall prevalence of first line ART treatment failure identified in this study showed that 15.3\% (95\% CI: 12, 18.6) ART user patients faced first line treatment failure in Ethiopia. The result of this meta-analysis is in line with that of a study conducted in Haiti (15\%) [39], higher than that of studies in Burkina Faso (6.4\%), Ghana (6.5\%), and Tanzania (7\%) [40-42] and lower than the result in Uganda (34\%) [43]. The possible explanation for the observed variations could be attributed to methodological difference in the assessment of failure, sample size, socio-economic, and medical service that has a great impact on treatment failure detection.

We did subgroup analysis due to a significant heterogeneity as shown in (Fig. 2) which indicated the highest prevalence of treatment failure was immunological (11.5\%), followed by clinical failure (6.7\%), whereas the lowest was virological $(5.8 \%)$. The reason behind is

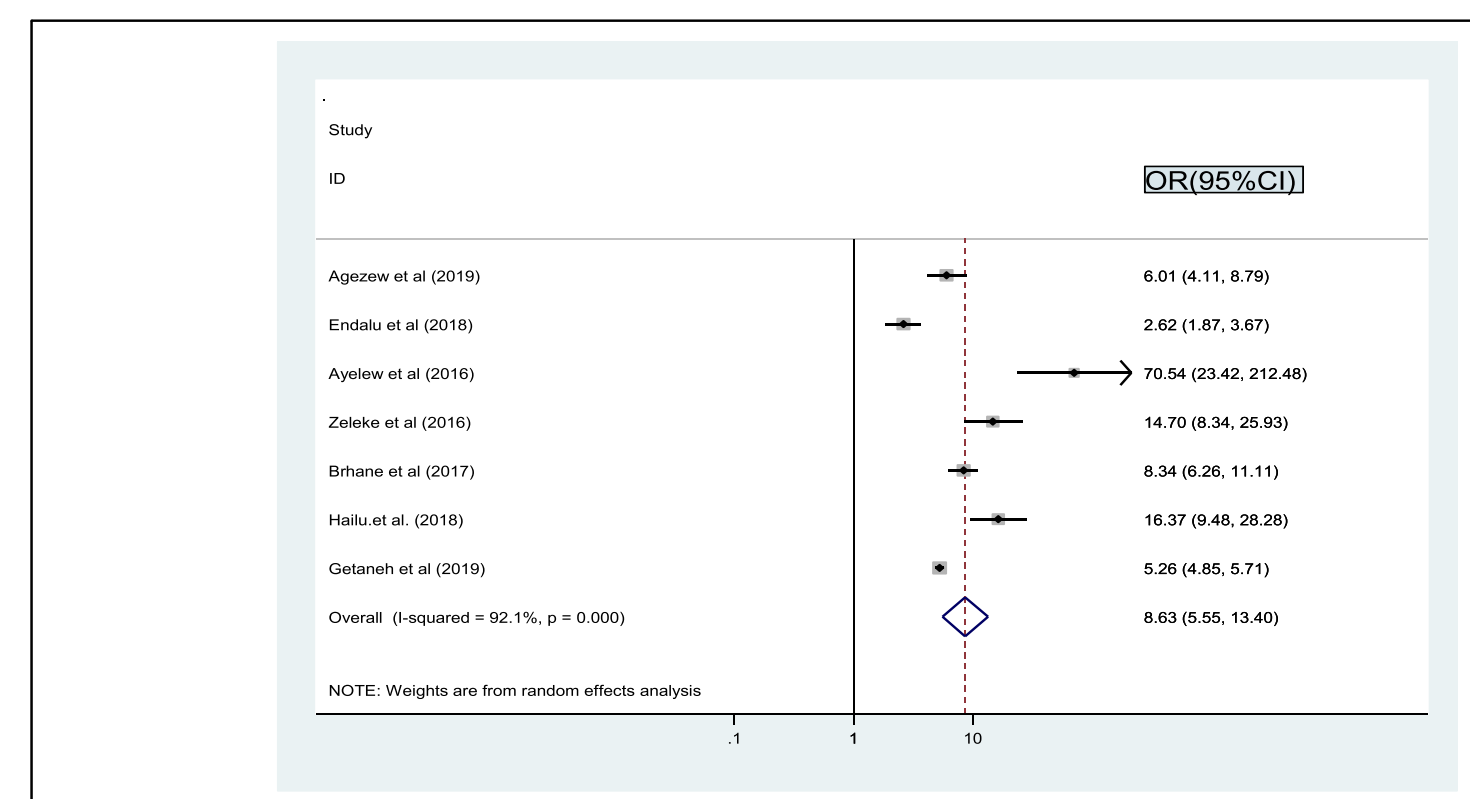

Fig. 3 Pooled odd ratio between adherence and treatment failure 


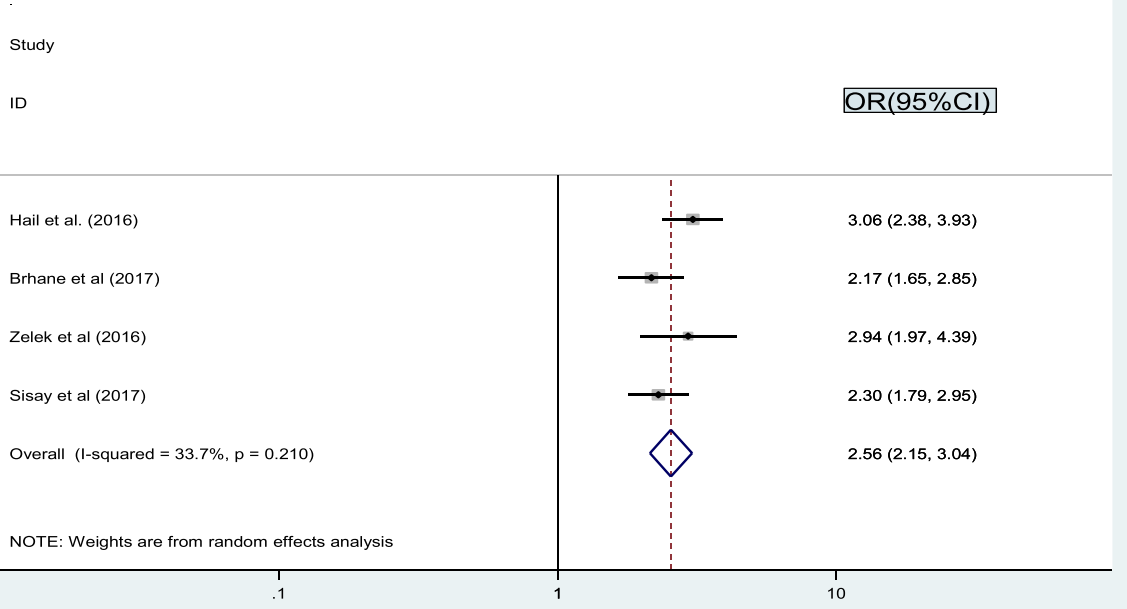

Fig. 4 Pooled odd ratio between co-infection and treatment failure

clinical and immunological criteria were found to perform relatively poor in predicting virological failure of ART [44]. The possible explanation for this variation could be that immunological and clinical criteria have poor sensitivity and positive predictive value to detect treatment failure, particularly immunological failure relying on higher $\mathrm{CD} 4$ cell counts for treatment monitoring would therefore lead to misclassifications of treatment failure [45-48]. In addition, regular virological treatment failure detection is low because of inadequate capacity and laboratory facilities in Ethiopia.

Likewise, the subgroup analysis of this study on mean sample size with below and above the mean but both indicated almost equal to the overall pooled prevalence with 15 and $16.3 \%$ respectively. The possible explanations for this similarity could be due to the mean samples size are sufficiently large. Consequently, the larger sample increases the estimation of parameters both above and below mean.

The region of the country, type of failure assessment criteria, study population, publication year and sample sizes were investigated by using univariate metaregression models, but none of these variables were found to be statistically significant. Publication bias using Egger's tests did not show statistical significant for estimating the prevalence of treatment failure among ART users with $p=0.80$.

This meta-analysis also aims to identify the possible determinant factors on the magnitude of treatment failure among HIV patients in Ethiopia. In this study,

Study

$\mathrm{OR}(95 \% \mathrm{Cl})$

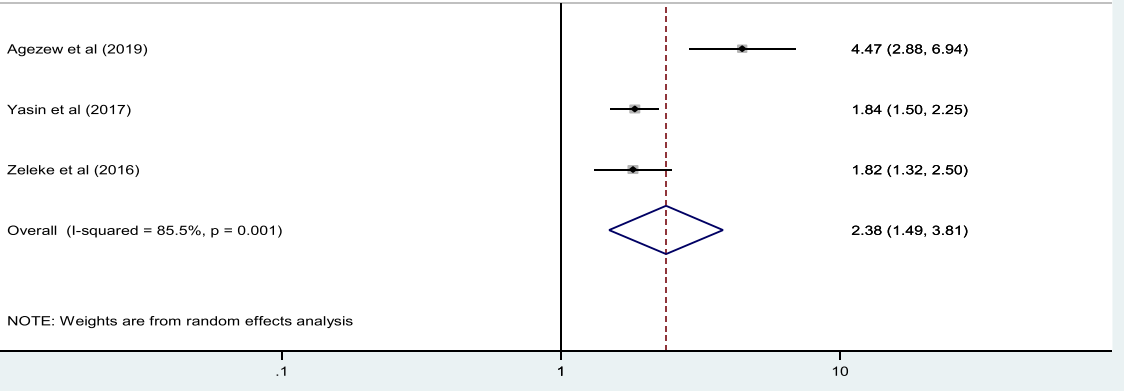

Fig. 5 Pooled odd ratio between advanced WHO clinical stage and treatment failure 


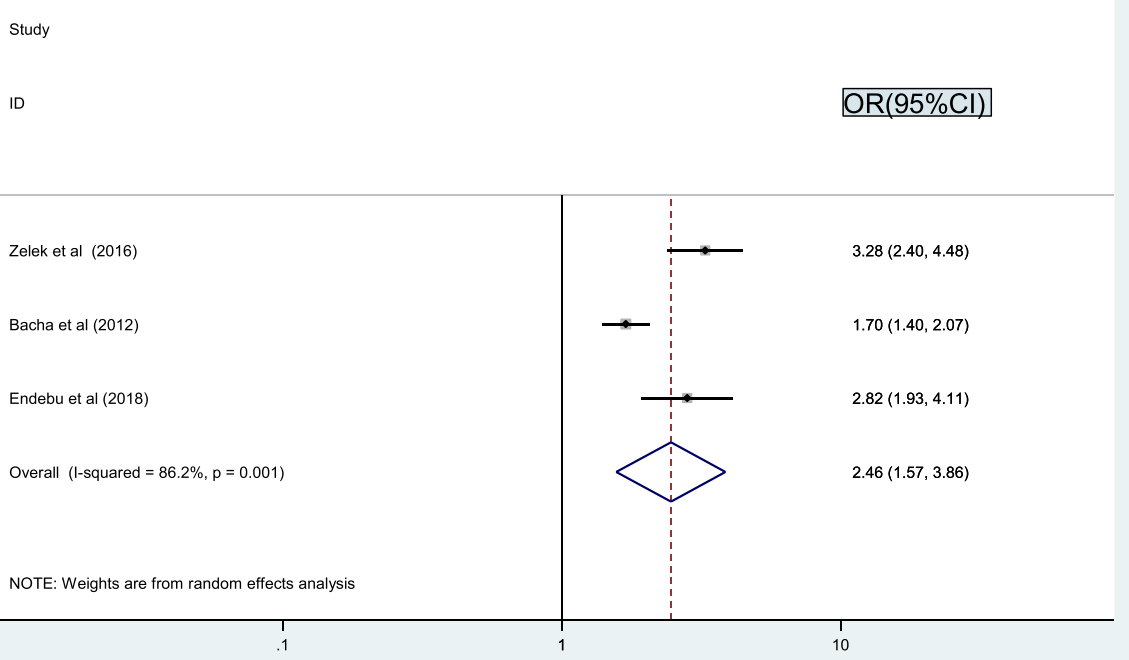

Fig. 6 Pooled odd ratio between regimen change and treatment failure

adherence, co-infection, advanced WHO clinical stage, disclosure, and regimen change were found statistically associated factors for treatment failure. The success of ART reflects, in part, patient adherence to treatment and the present finding revealed that adherence is significantly indication of treatment failure [49]. Accordingly, the probability of treatment failure was 8.6 times higher among patients who did not use ART continuously Poor adherence is one of the ways in which drug adaptability and resistance developed [50].

Disclosure is another determinant factor for treatment failure. In line with study in Tanzania, this study showed that not disclose HIV infection status was two times higher risk of treatment failure [50]. Basically nondisclosure leads to 'hidden behaviors' and potentiates nonadherence as a cause for treatment failure. However, one study conducted Ethiopia, showed non-disclosure as a protective factor for treatment failure due to stigma and discrimination [17].

In addition, co-infection is an associated factor of treatment failure among patients on ART. As supported by studies in South Africa and Uganda, co-infection was a determinant factor for ART treatment failure [51, 52]. This could be due to having advanced opportunistic

Study

ID

$\mathrm{OR}(95 \% \mathrm{Cl})$

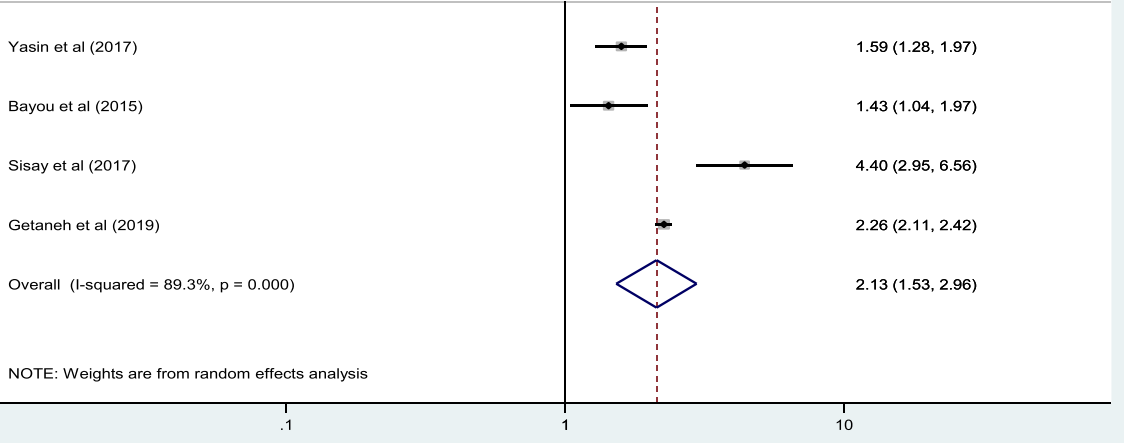

Fig. 7 Pooled odd ratio between disclosure and treatment failure 
infection/co-infection which may deplete CD4 counts and compromise immunity and may negatively affect response to treatment. In addition, medications for coinfection treatment and ART together can contribute to double burden side effects.

Pooled prevalence of treatment failure was associated with regimen change. Regimen change yield a 2.5 times higher possibility of developing treatment failure which is similar with studies conducted in Myanmar and Malawi [53, 54].

Finally, advanced WHO clinical stage was a significant predictor of ART treatment failure as indicated in studies in South Africa and Uganda [55, 56]. The likelihood of treatment failure was 2.4 times higher among patients in advanced clinical stage III/IV as compared to stage I/ II in this meta-analysis. This finding could be due to advanced HIV disease which is often associated with loss to follow-up [57].

Even though the study is nationally based; including only English articles and having a relatively small sample size was the limitation of the study. In addition this metaanalysis represented only studies reported from three regions and two administrative town of the country, which could bias the estimate of treatment failure proportion for the entire Ethiopian context. Furthermore, the included studies did not incorporated a number of factors such as age, sex, duration of follow up, opportunistic infection, and body mass index as a possible factor to examine the pooled odds ratios.

\section{Conclusion}

In this study, treatment failure among ART users in Ethiopia was significantly high. Adherence, co-infection, advanced WHO clinical stage, regimen change and disclosure are determinant factors for treatment failure. Therefore, improved drug adherence, prevention of coinfection, as well as timely and rigorous follow up, were found to sustain first-line therapy effectiveness and prevent HIV-drug resistance.

\section{Abbreviations}

AIDS: Acquired immune deficiency syndrome; ART: Antiretroviral therapy; HAART: Highly active antiretroviral therapy; HIV : Human immune virus; PRISMA: Preferred Reporting Items for Systematic Reviews and Meta-Analysis; WHO: World Health Organization

\section{Acknowledgements}

Authors of primary study.

\section{Authors' contributions}

MAA: Conception of research protocol, study design, literature review, data extraction, data analysis, interpretation and drafting the manuscript. MA and DBK contribute on data extraction, quality assessment, data analysis and manuscript review and SM edition \& validation. All authors have read and approved the manuscript.

\section{Funding}

Not applicable
Availability of data and materials

Minimal data could be accessed upon request from first author (MAA).

Ethics approval and consent to participate

Not applicable

Consent for publication

Not applicable

\section{Competing interests}

The authors declare that they have no competing interests.

\section{Author details}

${ }^{1}$ Department of Public Health, College of Health Science Debre Markos University, P.O. Box 269, Debre Markos, Ethiopia. ${ }^{2}$ Department of Nursing, School of Health Science Bahir Dar University, Bahir Dar, Ethiopia.

Received: 9 August 2019 Accepted: 9 September 2019

Published online: 30 October 2019

\section{References}

1. Awoke DH, Tekebash A, Kidane A. Patterns of mortality in public and private hospitals of Addis Ababa, Ethiopia. BMC Public Health. 2012;12:1007.

2. Max Roser HR. Burden of Disease in our World in data is free and accessible for everyone; 2018.

3. Joint United Nations Program on HIV/AIDS. Global report. Geneva: UNAIDS report on the global AIDS epidemic. 2017.

4. UNAIDS, WHO. Global update on HIV treatment: WHO; 2017.

5. UNAIDS. Global AIDS monitoring 2017: Indicators for monitoring the 2016 United Nations Political Declaration on HIV and AIDS. Geneva: UNAIDS; 2017

6. HIV/AIDS Strategic Plan 2015-2020 by the Federal Democratic Republic of Ethiopia Ministry of Health, Federal HIV/AIDS Prevention and Control Office Addis Ababa, Ethiopia. 2014.

7. HIV/AIDS Glossary. https://aidsinfo.nih.gov/understanding-hivaids/glossary/ 873/treatment-failure.

8. Ethiopian consolidated guidelines on the use of antiretroviral drugs for treating and preventing HIV infection. 2013.

9. Estill FN, Salazar-Vizcaya L, Haas A, Blaser N, Egger M et al.. The estimated need of second-line antiretroviral therapy in sub-Saharan Africa 2015-2030: mathematical modelling study. 8th IAS conference on HIV pathogenesis, treatment \& prevention, Vancouver, Canada. 2015.

10. Mutevedzi PC, Rodger AJ, Newell ML. Association of age with mortality and virological and immunological response to antiretroviral therapy in rural South African adults. PLoS One. 2011:6:e2179.

11. Petersen ML, Napravnik S, Eron JJ, Moore RD, et al. Long-term consequences of the delay between virologic failure of highly active antiretroviral therapy and regimen modification. AIDS. 2008;22:2097.

12. World Health Organization (WHO). Towards universal access: Scaling up priority HIV/AIDS interventions in the health sector. Prog Rep. 2008;2008:1-77.

13. El-Khatib Z, Katzenstein D, Marrone G, Laher F, Mohapi L, et al. Adherence to drug-refill is a useful early warning indicator of virologic and immunologic failure among HIV patients on first-line ART in South Africa. PLOS One. 2011:6:e17518.

14. Ethiopian consolidated guidelines on the use of antiretroviral drugs for treating and preventing HIV infection. 2018.

15. Assefa A, Gebeyaw BG, Yitayew SG, et al. The effect of incident tuberculosis on immunological response of HIV patients on highly active anti-retroviral therapy at the university of Gondar hospital, northwest Ethiopia: a retrospective follow-up study. BMC Infect Dis. 2014;14:468.

16. Zeleke A. Prevalence of antiretroviral treatment failure and associated factors in HIV infected children on antiretroviral therapy at Gondar University Hospital, retrospective cohort study. Int J Med Med Sci. 2016:8:125.

17. Ayalew M. First-line antiretroviral treatment failure and associated factors in HIV patients at the University of Gondar Teaching Hospital, Gondar, Northwest Ethiopia. Dovepress HIV/AIDS Res Palliat Care. 2016;8:141.

18. Bekelech B, Kumie A. Assessment of the magnitude and associated factors of immunological failure among adult and adolescent HIV-infected patients in St. Luke and Tulubolo Hospital, Oromia Region, Ethiopia. Pan Afr Med J. 2015;21:291. 
19. Brhane BG, Abay GK. HIV/AIDS Treatment Failure and its Determinant Factors among First Line HAART Patients at Felege-Hiwot Referral Hospital, Bahir Dar, Northwest Ethiopia. J AIDS Clin Res. 2017;8:2.

20. Sisay C, Sisay A, Mekonen H, Terfa K, et al. Incidence and Predictors of Anti-Retroviral Treatment (ART) Failure among Adults Receiving HIV Care at Zewditu Memorial Hospital, Addis Ababa, Ethiopia. J AIDS Clin Res. 2017:8:2.

21. Haile D, Gashaw K, Demelash H, et al. Predictors of Treatment Failure among Adult Antiretroviral Treatment (ART) Clients in Bale Zone Hospitals, South Eastern Ethiopia. PLOS ONE. 2016;11:e0164299.

22. Genet GH, Dawit GH, Amlsha KH, Wasihun AG, Dejene T. Virological and immunological failure of HAART and associated risk factors among adults and adolescents in the Tigray region of Northern Ethiopia. PLoS ONE. 2018;13:e0196259.

23. Sisay MM, Ayele TA, Gelaw YA. Incidence and risk factors of first-line antiretroviral treatment failure among human immunodeficiency virus-infected children in Amhara regional state, Ethiopia: a retrospective follow-up study BMJ Open. 2017.

24. Agezew T, Tadesse A, Derseh L, Yimer M. Incidence and predictors of first line anti-retroviral therapy failure among adults receiving HIV care in North West Ethiopia: a hospital-based follow-up study. Infect Dis Epidemiol. 2019.

25. Bacha T, Birkneh T, Worku A, et al. Predictors of treatment failure and time to detection and switching in HIV-infected Ethiopian children receiving first line anti-retroviral therapy. BMC Infect Dis. 2012;12:197.

26. Endebu T, Deksisa A, Moges T, Kisi T, Ensermu T. Incidence of virological failure and associated factors among adult HIV-positive patients on first line antiretroviral therapy regimen, Central Ethiopia. Int J HIV/AIDS Prev Educ Behav Sci. 2018.

27. Yimer TY. Magnitude and Predictors of Anti-Retroviral Treatment (ART) failure in private health facilities in Addis Ababa, Ethiopia. PLoS ONE. 2015; 10:e0126026.

28. Teshome W, Tefera A. Detection of immunological treatment failure among HIV infected patients in Ethiopia: a retrospective cohort study. BMC Immunol. 2015;16:55

29. Assefa $Y$, Lynen L, Kloos H, et al. Brief report: long-term Outcomes and their determinants in patients on antiretroviral treatment in Ethiopia, 2005/62011/12: a retrospective cohort study. JAIDS J Acquir Immune Defic Syndr. 2015:70(4):414-9.

30. Melsew YA, Tessema GA, Ayele T. Rate of immunological failure and its predictors among patients on highly active antiretroviral therapy at Debremarkos Hospital, Northwest Ethiopia: a retrospective follow up study. J AIDS Clin Res. 2013.

31. YG. Atsbeha, K. Zealiyas, R. Tilahun, M. Girma, G.G Michael, T. Deressa, et al. Treatment failure among people living with HIV taking antiretroviral therapy in Ethiopia. bioRxiv preprint first posted online. 2019.

32. Liberati AD, Tetzlaff J, Mulrow C, et al. The PRISMA statement for reporting systematic reviews and meta-analyses of studies that evaluate health care interventions: explanation and elaboration. J Clin Epidemiol. 2009;62:e1.

33. NEWCASTLE -OTTAWA QUALITY ASSESSMENT SCALE (adapted for cross sectionalstudies).

34. Rücker SG, Carpenter JR, Schumacher M. Undue reliance on I 2 in assessing heterogeneity may mislead. BMC Med Res Methodol. 2008;8:79.

35. Nyaga VN, Arbyn M, Aerts M. Metaprop: a Stata command to perform metaanalysis of binomial data. Arch Public Health. 2014;72:39.

36. Borenstein M, Hedges L, Higgins J, Rothstein HR. A basic introduction to fixed-effect and randomeffects models for meta-analysis. Res Synth Methods. 2010;1:97.

37. Yassin S, Gebretekle G. Magnitude and predictors of antiretroviral treatment failure among HIV-infected children in Fiche and Kuyu hospitals, Oromia region, Ethiopia: a retrospective cohort study. Pharmacol Res Perspect. 2017; 5:e00296.

38. Yirdaw KD, Hattingh S. Prevalence and predictors of immunological failure among HIV patients on HAART in Southern Ethiopia. PLOS One. 2015;10:e0125826

39. Charles M, Severe P, Guiteau C, Apollon A. Virologic, clinical and immunologic responses following failure of first-line antiretroviral therapy in Haiti. J Int AIDS Soc. 2012;15:17375.

40. Vanobberghen FM, Kilama B, Alison W, et al. Immunological failure of firstline and switch to second-line antiretroviral therapy among HIVinfected persons in Tanzania: analysis of routinely collected national data. Tropical Med Int Health. 2015;20:880.
41. Poda ZA, Sondo KA, Diallo e. Prevalence and factors associated with treatment failure during antiretroviral therapy Atbobo-Dioulasso University Teaching Hospital (Burkina Faso) (2008-2013). Monroe Township: Austin Publishing Group; 2016.

42. Kukoyi O, LR JP, et al. Viral load monitoring and antiretroviral treatment outcomes in a pediatric HIV cohort in Ghana. BMC Infect Dis. 2016.

43. Sebunya R, Musiime V, Kitaka SB, et al. Incidence and risk factors for first line anti retroviral treatment failure among Ugandan children attending an urban HIV clinic. AIDS Res Ther. 2013;10:25.

44. Ferreyra C, Yun O, Eisenberg N, Alonso E, Khamadi AS, Mwau M, et al. Evaluation of clinical and immunological markers for predicting virological failure in a HIV/ AIDS treatment cohort in Busia, Kenya. PLoS One. 2012;7:e49834.

45. Rutherford GW, Anglemyer A, Easterbrook PJ, Horvath T, et al. Predicting treatment failure in adults and children on antiretroviral therapy: a systematic review of the performance characteristics of the 2010 WHO immunologic and clinical criteria for virologic failure. AIDS. 2014;28:S161.

46. Reynolds SJ, Nakigozi G, Newell K, Ndyanabo A, Galiwongo R, Boaz I, et al. Failure of immunologic criteria to appropriately identify antiretroviral treatment failure in Uganda. AIDS. 2009;23:697.

47. Mee P, Fielding K, Charalambous S, Churchyard GJ, Grant A. Evaluation of the WHO criteria for antiretroviral treatment failure among adults in South Africa. AIDS. 2008;22:1971.

48. Daniel W, Benson R, Kidenya SE, Semvua B, et al. Accuracy of WHO immunological criteria in identifying virological failure among HIV-infected adults on First line antiretroviral therapy in Mwanza, North-western Tanzania. BMC Res Notes. 2017;10:45.

49. Ugwu R, Eneh A. Factors influencing adherence to pediatric antiretroviral therapy in Portharcourt, South- South Nigeria. Pan Afr Med J. 2013;16:30

50. Ramadhani NT, Landman KZ, Ndosi EM, Gao F, Kirchherr JL, et al. Predictors of incomplete adherence, virologic failure, and antiviral drug resistance among HIV-infected adults receiving antiretroviral therapy in Tanzania. Clin Infect Dis. 2007:45:1492-8.

51. Eshun-Wilson I, Taljaard J, Nachega JB. Sub-optimal CD4 T-lymphocyte responses among HIV infected patients who develop TB during the first year of ART. AIDS Clin Res. 2012.

52. Kiragga AN, Schaefer $P$, Kambugu A, Hoepelman A, Manabe YC. Incident tuberculosis during antiretroviral therapy contributes to suboptimal immune reconstitution in a large urban HIV clinic in sub-Saharan Africa. PLoS One. 2010;5:e10527.

53. Buck WC, Kabue MM, Kazembe PN, Kline MW. Discontinuation of standard first-line antiretroviral therapy in a cohort of 1434 Malawian children. J Int AIDS Soc. 2010;13:31.

54. Kyaw NT, Harries AD, Kumar AM, Kyaw WY, Win T. High rate of virological failure and low rate of switching to second-line treatment among adolescents and adults living with HIV on first-line ART in Myanmar, 20052015. PLOS ONE. 2017.

55. Fox MP, Cutsem GV, Giddy J, Maskew M, Keiser O, et al. Rates and predictors of failure of first-line antiretroviral therapy and switch to second-line ART in South Africa. J Acquir Immune Defic Syndr. 2012.

56. Kazooba P, Mayanja BN, Levin J, Masiira B, Kaleebu P. Virological failure on first-line antiretroviral therapy; associated factors and a pragmatic approach for switching to second line therapy-evidence from a prospective cohort study in rural South-Western Uganda, 2004-2011. Pan Afr Med J. 2018;29:1.

57. Assemie MA, Muchie KM, Ayele TA. Incidence and predictors of loss to follow up among HIV-infected adults at Pawi General Hospital, northwest Ethiopia: competing risk regression model. BMC Res Notes. 2018;11:287.

Ready to submit your research? Choose BMC and benefit from:

- fast, convenient online submission

- thorough peer review by experienced researchers in your field

- rapid publication on acceptance

- support for research data, including large and complex data types

- gold Open Access which fosters wider collaboration and increased citations

- maximum visibility for your research: over $100 \mathrm{M}$ website views per year

At $\mathrm{BMC}$, research is always in progress.

Learn more biomedcentral.com/submission 